\title{
Brain-derived neurotrophic factor inhibits hyperglycemia-induced apoptosis and downregulation of synaptic plasticity-related proteins in hippocampal neurons via the PI3K/Akt pathway
}

\author{
YUAN ZHONG, YITONG ZHU, TING HE, WEI LI, QINJIE LI and YA MIAO \\ Department of Gerontology, Shanghai Jiao Tong University Affiliated Sixth People's Hospital, Shanghai 200233, P.R. China
}

Received April 29, 2018; Accepted October 4, 2018

DOI: $10.3892 /$ ijmm.2018.3933

\begin{abstract}
It is not known whether brain-derived neurotrophic factor (BDNF) protects hippocampal neurons from high glucose-induced apoptosis and/or synaptic plasticity dysfunction. The present study aimed to assess whether BDNF exerted a neuroprotective effect in rat hippocampal neurons exposed to high glucose and examine the underlying mechanisms. The apoptosis of primary hippocampal neurons was assessed by Annexin V-fluorescein is othiocyanate/propidium iodide staining. The mRNA and protein expression levels were measured by reverse transcription-quantitative polymerase chain reaction and western blot experiments, respectively. Synaptic plasticity was evaluated by the immunolocalization of synaptophysin (Syn). Exposure of the hippocampal neurons to high glucose $(75 \mathrm{mM}$ for $72 \mathrm{~h}$ ) resulted in cell apoptosis, decreased mRNA and protein expression levels of three synaptic plasticity-related proteins (Syn, Arc and cyclic AMP response element-binding protein), and changes in the cellular distribution of Syn, indicating loss of synaptic density. These effects of high glucose were partially or completely reversed by prior administration of BDNF (50 ng/ml for $24 \mathrm{~h}$ ). Pre-treatment with wortmannin, a phosphatidylinositol-3-kinase (PI3K) inhibitor, suppressed the ability of BDNF to inhibit the effects of high glucose. In addition, BDNF significantly upregulated the tropomyosin-related kinase B, its cognate receptor, Akt and phosphorylated Akt at the protein levels under high glucose conditions. In conclusion, high glucose induced apoptosis and downregulated synaptic plasticity-related proteins in hippocampal neurons. These effects were reversed by BDNF via the PI3K/Akt signaling pathway.
\end{abstract}

Correspondence to: Dr Ya Miao, Department of Gerontology, Shanghai Jiao Tong University Affiliated Sixth People's Hospital, 600 Yishan Road, Xuhui, Shanghai 200233, P.R. China

E-mail: nning-my@163.com

Key words: brain-derived neurotrophic factor, rat hippocampal neurons, hyperglycemia, apoptosis, synaptic plasticity

\section{Introduction}

Diabetes mellitus is estimated to affect $>366,000,000$ individuals worldwide and is characterized by chronic hyperglycemia (1). Studies in humans and animal models have reported an association between diabetes and neurological conditions that affect learning and memory, including Alzheimer's disease (AD) (2-4). Diabetic encephalopathy is now recognized as a complication of diabetes (5). Hyperglycemia has been shown to significantly decrease cell viability and induce apoptosis and loss of hippocampal neurons. The effect of high glucose accumulation involves the intracellular accrual of reactive oxygen species (ROS) $(6,7)$. Therefore, it is necessary to develop neuroprotective strategies to inhibit diabetic encephalopathy. One avenue of investigation has focused on neurotrophic factors, which are important for neuronal survival and regeneration and are considered potential therapeutics for $\mathrm{AD}$ and other neurodegenerative diseases (8).

Brain-derived neurotrophic factor (BDNF) is a specific neurotrophic factor that is expressed in neurons and is involved in the growth and differentiation of new neurons and synapse development. BDNF binds to two receptors, namely tropomyosin-related kinase B (TrkB) and low-affinity nerve growth factor receptor, and is involved in the process of long-term memory. BDNF provides trophic support to neurons and exerts a neuroprotective effect against brain injury. In addition to its well-established role in the survival, differentiation and plasticity of neurons (9), BDNF and its cognate receptor TrkB are implicated in the regulation of energy and glucose homeostasis through their effects on the central nervous system (10). Perturbed BDNF signaling in the brain triggers hyperphagia and obesity in mice, suggesting that BDNF acts as an anorexigenic signaling factor (10). Studies have suggested that BDNF regulates glucose metabolism by improving insulin sensitivity and increasing pancreatic insulin production $(11,12)$. In addition, in rodents with impaired leptin signaling through diet-induced obesity and/or deficient leptin signaling (db/db mice), systemic or central BDNF administration has been shown to reduce food intake and body weight (11) in a dose-dependent manner (13).

Synaptic plasticity is defined as the ability of synapses to reinforce and/or weaken their connections over time, depending on their relative activity levels. Synaptic plasticity is considered as one of the most important neurochemical 
processes involved in learning and memory (14). In animals, diabetes can cause changes in synaptic proteins, and hyperglycemia is one of the factors contributing to these alterations (15). Synaptophysin (Syn) is a key protein involved in the biogenesis and exo-endocytosis of synaptic vesicles. Syn is considered to be a specific marker of synaptic density and is closely associated with activity-dependent synapse formation and synaptic plasticity $(16,17)$. Syn is mainly degraded through the ubiquitin-proteasome system (18), and evidence suggests that reduced expression levels of Syn may contribute to hyperglycemia-induced cognitive impairment in mice (19). Other factors have also been implicated in synaptic plasticity and memory. Cyclic AMP response element-binding protein (CREB) is a nuclear transcription factor that is essential for the formation and retention of memory. The activation of CREB occurs by phosphorylation at serine residue 133 and is required for neuronal growth and survival (20). CREB is necessary for the maintenance of normal synapses in hippocampal neurons (21), and reduced levels of phosphorylated CREB (p-CREB) have been observed in the postmortem brains of patients with AD and experimental models of AD $(22,23)$. Arc protein, which is transcribed from the Arc/Arg3.1 gene, is another factor associated with the progression of AD (24-26). The dysregulation of Arc in cerebral neurons interferes with their normal activity and causes synaptic damage and neuron loss, leading to the degradation of specific neural circuit functions and a decrease in neuronal network activity that may be involved in AD (24-26).

Previous studies have investigated the interaction of the TrkB receptor with hyperglycemia and neuronal function $(27,28)$. BDNF upregulates TrkB and increases the phosphorylation levels of TrkB and ERK in retinal neurons exposed to hyperglycemic conditions (29). A previous study demonstrated that the phosphorylation levels of the cell signaling molecule Akt (protein kinase B) and transcription factor CREB are reduced in diabetic rats compared with those in control animals (30), suggesting that these factors may be involved in diabetes-induced cognitive dysfunction. Chen et al (31) demonstrated that the neuroprotective effects of BDNF, acting via the TrkB receptor, were induced by activation of the phosphatidylinositol-3-kinase (PI3K)-Akt pathway and the increased expression of Arc.

However, whether BDNF protects hippocampal neurons from high glucose-induced apoptosis and/or synaptic plasticity dysfunction remains to be fully elucidated. Therefore, the aim of the present study was to evaluate whether long-term elevated glucose, which mimics prolonged hyperglycemia, causes significant changes in neuronal survival and synaptic plasticity, and whether exogenous BDNF exerts neuroprotective effects.

\section{Materials and methods}

Primary culture of rat hippocampal neurons. All animal experiments were performed in accordance with the National Institutes of Health Guidelines for the Care and Use of Laboratory Animals and approved by the Ethics Committee of Animal Experiments of the Shanghai Sixth People's Hospital affiliated to Shanghai Jiao Tong University [Shanghai, China; permit no. SYXK (Shanghai) 2011-0128].
Primary cultures of rat hippocampal neurons were prepared from the hippocampi of 10 neonatal Sprague-Dawley rats within $24 \mathrm{~h}$ of birth (Shanghai Laboratory Animal Co., Ltd., Shanghai, China), weighing between 4.5-6.5 g, as described previously (32), with minor modifications. The hippocampi were dissected from the rat brain tissues and were placed on ice. Subsequently, the blood vessels and meninges were thoroughly removed, and the hippocampi were washed with phosphate-buffered saline (PBS). The tissues were then transferred into Eppendorf tubes containing $1 \mathrm{ml} 0.123 \%$ trypsin (Gibco; Thermo Fisher Scientific, Inc., Waltham, MA, USA). The hippocampi were cut into small pieces with sterile ophthalmic scissors (Kun Sheng Medical Instrument Co., Ltd., Shanghai, China). The hippocampal pieces were digested for $15 \mathrm{~min}$ at $37^{\circ} \mathrm{C}$ with vortexing every $5 \mathrm{~min}$. The digestion procedure was terminated by the addition of $5 \mathrm{ml}$ Dulbecco's modified Eagle's medium (DMEM; Gibco; Thermo Fisher Scientific, Inc.) containing 20\% fetal bovine serum (FBS; Gibco; Thermo Fisher Scientific, Inc.). The cell suspension was passed through a 200-mesh cell strainer and separated by centrifugation at $300 \times \mathrm{g}$ for $5 \mathrm{~min}$ at room temperature. The pellets were resuspended in $2 \mathrm{ml}$ of DMEM containing $20 \% \mathrm{FBS}$, at $\sim 70$ cells per $\mathrm{ml}$. The neurons were seeded on poly-D-lysine $(0.1 \mathrm{mg} / \mathrm{ml}$; Gibco; Thermo Fisher Scientific, Inc.)-coated glass coverslips (Corning Incorporated, Corning, NY, USA), 96-well plates and/or 6-well plates in 60-70 $\mu \mathrm{l}$ medium. Following 6-12 h of incubation, the cells were cultured in Neurobasal medium supplemented with B27 (1:50, Gibco; Thermo Fisher Scientific, Inc.). Half of the medium was replaced with fresh medium every 2 or 3 days.

Immunofluorescence staining. The primary hippocampal neurons were fixed with $4 \%$ paraformaldehyde (China National Medicines Corporation, Ltd., Beijing, China) for $1 \mathrm{~h}$ at room temperature and incubated in PBS containing $0.5 \%$ Triton for $20 \mathrm{~min}$ at room temperature. Non-specific antibody binding was blocked by incubation at room temperature for 30 min with normal goat serum (Gibco; Thermo Fisher Scientific, Inc.). Each coverslip was incubated with $20 \mu 1$ rabbit anti-NeuN primary antibody (1:200; cat. no. ab177487, Abcam, Cambridge, MA, USA) or rabbit anti-synaptophysin primary antibody (1:200; cat. no. ab32127; Abcam) at $4^{\circ} \mathrm{C}$ overnight. The coverslips were subsequently washed three times in PBS and incubated with donkey anti-rabbit secondary antibody (1:500; cat. no. A0453, Alex fluor 555; Beyotime Institute of Biotechnology, Shanghai, China) or goat anti-rabbit secondary antibody (1:500; cat. no. A0423; Alex fluor 488; Beyotime Institute of Biotechnology) for $30 \mathrm{~min}$ at room temperature. The coverslips were finally incubated with $20 \mu \mathrm{l}$ of 4',6-diamidino-2-phenylindole (DAPI; Roche Diagnostics, Basel, Switzerland) for $5 \mathrm{~min}$ at room temperature in the dark. The cells were observed and images were captured using a Volocity Demo imaging system (PerkinElmer, Inc., Waltham, MA, USA).

High glucose exposure and experimental grouping. The hippocampal neurons in primary culture for 3 days were divided into four experimental groups, including the control group (CON), high glucose group $(\mathrm{HG})$, high glucose $+\mathrm{BDNF}$ group $(\mathrm{HG}+\mathrm{BDNF})$ and high glucose + BDNF + wortmanin 
Table I. Primer sequences for reverse transcription-quantitative polymerase chain reaction.

\begin{tabular}{llcc}
\hline Gene & \multicolumn{1}{c}{ Primer sequence $(5 '-3 ')$} & Product length $(\mathrm{bp})$ & Temperature $\left({ }^{\circ} \mathrm{C}\right)$ \\
\hline GAPDH & F: CAGGGCTGCCTTCTCTTGTG & 111 & 60.70 \\
\multirow{2}{*}{ Arc } & R: AACTTGCCGTGGGTAGAGTC & & 60.54 \\
& F: TATGTGGACGCTGAGGAGGA & 77 & 60.77 \\
CREB & R: CGCAGAAAGCGCTTGAACTT & & 60.75 \\
\multirow{2}{*}{ Syn } & F: AGGGCCTGCAGACATTAACC & & 60.03 \\
& R: TGTCCATCAGTGGTCTGTGC & 78 & 60.04 \\
& F: TCGTGTTCAAGGAGACAGGC & & 60.80 \\
\end{tabular}

GAPDH, glyceraldehyde 3-phosphate dehydrogenase; CREB, cyclic AMP response element-binding protein; Syn, synaptophysin; F, forward; $\mathrm{R}$, reverse.

group (HG + BDNF + wort). The hippocampal neurons were seeded in 96-well plates at a density of 5,000-10,000 cells in each well and maintained at $37^{\circ} \mathrm{C}$ in a humidified incubator supplemented with $5 \% \mathrm{CO}_{2}$. Each of the four groups was exposed to different intervention measures. The control group was exposed to normal medium containing $25 \mathrm{mM}$ glucose. The primary hippocampal neurons were exposed to $75 \mathrm{mM}$ glucose (China National Medicines Corporation, Ltd.) for $72 \mathrm{~h}$ (33), which has no effect on normal metabolism. To establish the HG + BDNF group, the primary hippocampal neurons were incubated for $24 \mathrm{~h}$ with $50 \mathrm{ng} / \mathrm{ml}$ BDNF (Sigma; Merck KGaA, Darmstadt, Germany) prior to stimulation with $75 \mathrm{mM}$ glucose for $72 \mathrm{~h}$. Primary hippocampal neurons in the $\mathrm{HG}+\mathrm{BDNF}+$ wort group were pretreated with $0.5 \mu \mathrm{M}$ of wortmannin (Selleck Chemicals, Houston, TX, USA) to suppress PI3K for $2 \mathrm{~h}$, and further treatments were the same as those for the HG + BDNF group.

Assessment of apoptosis by flow cytometry. The apoptotic rate was measured using an Annexin V-fluorescein isothiocyanate (FITC)/propidium iodide (PI) apoptosis detection kit (Gibco; Thermo Fisher Scientific, Inc.). Flow cytometric data were acquired on FACSCalibur flow cytometer (BD Biosciences, San Jose, CA, USA) and analysed with the use of FlowJo v10 software (FlowJo, LLC, Ashland, OR, USA). Following $72 \mathrm{~h}$ of incubation, the primary hippocampal neurons were washed twice with ice-cold PBS and stained with $190 \mu 1$ Annexin V-FITC (Gibco; Thermo Fisher Scientific, Inc.) and $10 \mu 1$ PI (Roche Diagnostics) according to the manufacturer's protocol. Following $30 \mathrm{~min}$ of incubation at $37^{\circ} \mathrm{C}$, the stained neurons were analyzed by flow cytometry, and the rate of cellular apoptosis was determined. Annexin V was set as the horizontal axis and PI as the vertical axis. Apoptotic or necrotic cells were indicated in the upper right quadrant of the flow-cytogram, whereas early apoptotic cells were indicated in the lower right quadrant.

Reverse transcription-quantitative polymerase chain reaction $(R T-q P C R)$ analysis. Total RNA was isolated from the primary hippocampal neurons using TRIzol (Invitrogen; Thermo Fisher Scientific, Inc.). cDNA synthesis was performed at $37^{\circ} \mathrm{C}$ for
15 min followed by $85^{\circ} \mathrm{C}$ for $5 \mathrm{sec}$ using the PrimeScript ${ }^{\mathrm{TM}} \mathrm{RT}$ reagent kit (Takara Biotechnology Co., Ltd., Dalian, China). Specific mRNA quantification was performed by real-time PCR using SYBR ${ }^{\circledR}$ Premix Ex Taq ${ }^{\text {TM }}$ II (Tli RNase H Plus; Takara Biotechnology Co., Ltd.) in a FTC3000HT real-time PCR system (Funglyn Biotech, Inc., Toronto, ON, Canada). The gene-specific primers used are presented in Table I. Each PCR mixture contained $1 \mu \mathrm{l}$ cDNA, $0.8 \mu \mathrm{l}$ each primer

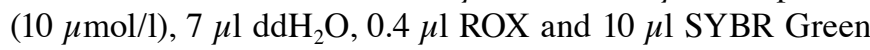
Premix (Takara Bio, Inc., Shiga, Japan). All reactions involved initial denaturation at $95^{\circ} \mathrm{C}$ for $30 \mathrm{sec}$ followed by 40 cycles of $95^{\circ} \mathrm{C}$ for $5 \mathrm{sec}$ and $60^{\circ} \mathrm{C}$ for $34 \mathrm{sec}$. The $2^{-\Delta \Delta \mathrm{Cq}}$ method (34) was used to determine the relative mRNA expression of the target genes. Glyceraldehyde 3-phosphate dehydrogenase (GAPDH) was used as an internal control.

Western blot analysis. The cells were lysed in radioimmunoprecipitation assay buffer (Beyotime Institute of Biotechnology) containing phenylmethylsulfonyl fluoride (final concentration $1 \mathrm{mM}$; Ameresco, Inc., Framingham, MA, USA) and centrifuged at $10,000 \mathrm{xg}$ for $5 \mathrm{~min}$ at $4^{\circ} \mathrm{C}$. The protein concentration was determined using a Bicinchoninic Acid Protein Assay kit (Beyotime Institute of Biotechnology). Equal quantities of protein $(60 \mu \mathrm{g})$ were separated by $10 \%$ sodium dodecyl sulfate-polyacrylamide gel electrophoresis and transferred onto polyvinylidene difluoride membranes (EMD Millipore, Burlington, MA, USA). The membranes were subsequently incubated in blocking buffer for $1 \mathrm{~h}, 5 \%$ bovine serum albumin (Gibco; Thermo Fisher Scientific, Inc.) in Tris-buffered saline with $0.1 \%$ Tween-20, followed by overnight incubation at $4^{\circ} \mathrm{C}$ with rabbit anti-CREB (1:1,000; cat. no. ab32515; Abcam), rabbit anti-Arc/Arg3.1 (1:1,000; cat. no. ab183183; Abcam), rabbit anti-TrkB (1:1,000; cat. no. 4603; Cell Signaling Technology, Inc.), rabbit anti-pAkt (1:1,000; cat. no. cst-4060s; Cell Signaling Technology, Inc.), rabbit anti-Akt (1:1,000; cat. no. cst-4691s; Cell Signaling Technology, Inc.), rabbit anti-Syn (1:5,000; cat. no. ab32127; Abcam) or mouse anti-GAPDH (1:1,000; cat. no. sc-293335; Santa Cruz Biotechnology, Inc., Dallas, TX, USA) antibodies. The membranes were subsequently incubated for $1 \mathrm{~h}$ at room temperature with goat anti-rabbit (1:5,000; cat. no. sc-2030) 
A

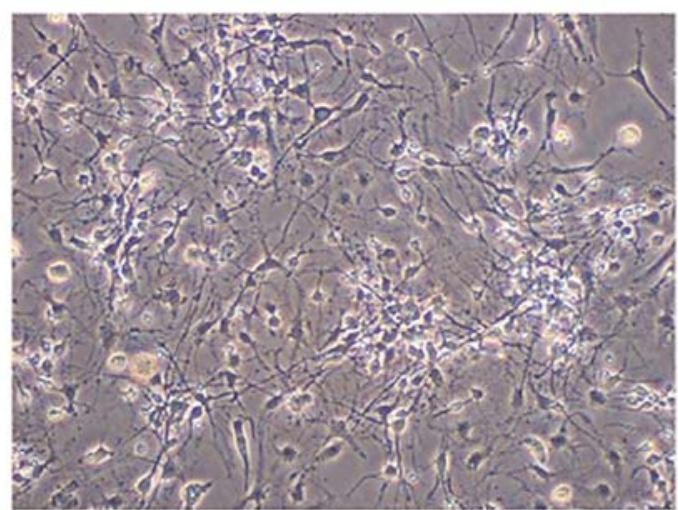

C

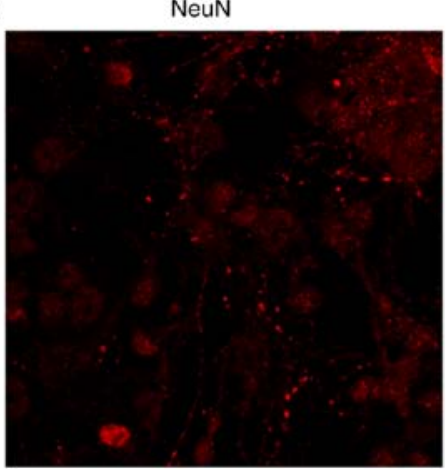

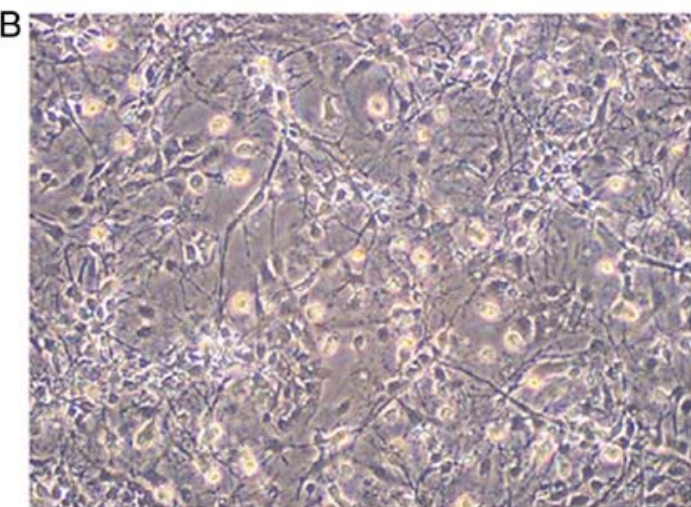

DAPI

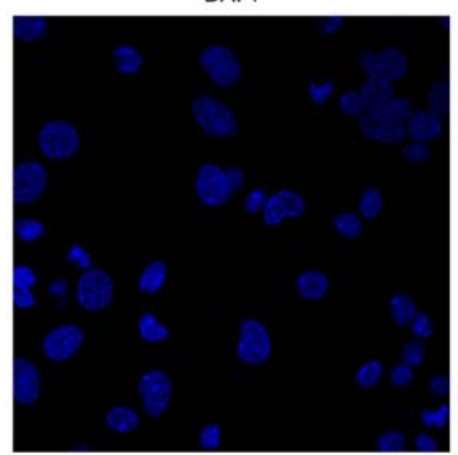

Merge

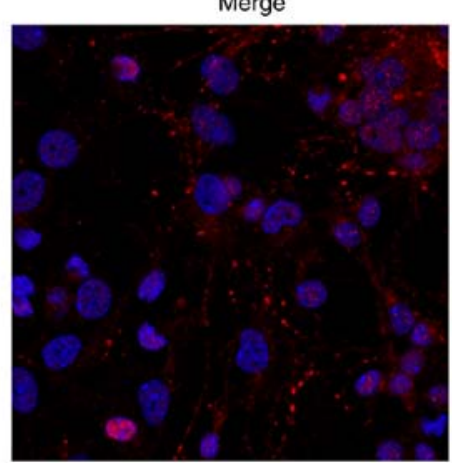

Figure 1. Identification of primary rat hippocampal neurons by immunofluorescence detection of NeuN protein. (A) Hippocampal neurons cultured for 3 days (magnification, x200). (B) Hippocampal neurons cultured for 5 days (magnification, x200). (C) Immunofluorescence detection of NeuN protein (red) and staining of the nuclei with DAPI (blue). The images were obtained by scanning laser confocal microscopy (magnification: $x 400)$. NeuN protein, neuron-specific nuclear protein; DAPI, 4',6-diamidino-2-phenylindole.

or anti-mouse (1:5,000; cat. no. sc-516180) IgG conjugated to horseradish peroxidase (Santa Cruz Biotechnology, Inc.). The detection of specific bands was achieved with enhanced chemiluminescence reagent (Pierce; Thermo Fisher Scientific, Inc.), and the immunoreactive bands were visualized on an ImageQuant LAS4000 mini apparatus (GE Healthcare, Chicago, IL, USA). Semi-quantification was performed using Image-Pro Plus v6.0 software (www.mediacy.com).

Statistical analysis. Statistical analyses were performed using Prism 5 software (GraphPad Software, Inc., La Jolla, CA, USA). Data are presented as the mean \pm standard deviation of three or four independent experiments. Statistical significance was determined by two-way analysis of variance followed by the Newman-Keuls post-hoc test. $\mathrm{P}<0.05$ was considered to indicate a statistically significant difference.

\section{Results}

Identification of hippocampal neurons. Previous studies have used hippocampal neurons from fetal tissues obtained from 18 days of pregnancy (35) or newborn rats $(36,37)$. Chen et al $(38)$ demonstrated no difference in the neuronal survival rates between hippocampal neurons from fetal rats and those from corresponding newborn rats. In the present study, hippocampal neurons from newborn rats were selected for culture in vitro. On days 3 and 5 of culture, the neurites were observed to interconnect with each other to form a loose network of cells (Fig. 1A and B), which is a typical function of cultured hippocampal neurons. Nuclear staining of the neurons was achieved using DAPI, and neurite growth was demonstrated by immunofluorescence staining of $\mathrm{NeuN}$ (red staining, Fig. 1C). The purity of the neurons, calculated as the ratio of the number of positive cells (identified by nuclear staining) to the total number of cells, was estimated to be $\sim 95 \%$ (Fig. 1C).

BDNF inhibits the high glucose-induced apoptosis of hippocampal neurons, and wortmannin reverses this effect. The apoptotic rate was significantly higher in hippocampal neurons treated with high glucose than in neurons exposed to normal glucose $(36.32 \pm 1.80$, vs. $2.68 \pm 0.60 \%, \mathrm{P}<0.001$; Fig. 2A and B). BDNF suppressed the apoptotic rate of neurons exposed to high glucose $(11.75 \pm 1.10$, vs. $36.32 \pm 1.80 \%$, $\mathrm{P}<0.001$; Fig. 2A and B). However, this effect of BDNF was attenuated by wortmannin, an inhibitor of PI3K (24.72 \pm 1.06 , vs. $11.75 \pm 1.10 \%, \mathrm{P}<0.01$; Fig. $2 \mathrm{~A}$ and $\mathrm{B})$. These data indicated that high glucose induced the apoptosis of hippocampal neurons cultured in vitro, which was suppressed by BDNF via PI3K signaling.

High glucose suppresses the expression levels of synaptic plasticity-related proteins, and BDNF reverses these effects. To examine the mechanism underlying the protective effect of BDNF on hippocampal neurons under hyperglycemic conditions, RT-qPCR and western blot experiments were performed to assess the expression levels of the synaptic plasticity-related proteins, CREB, Arc and Syn. The RT-qPCR experiments revealed that the mRNA expression levels of Syn, Arc and 
A
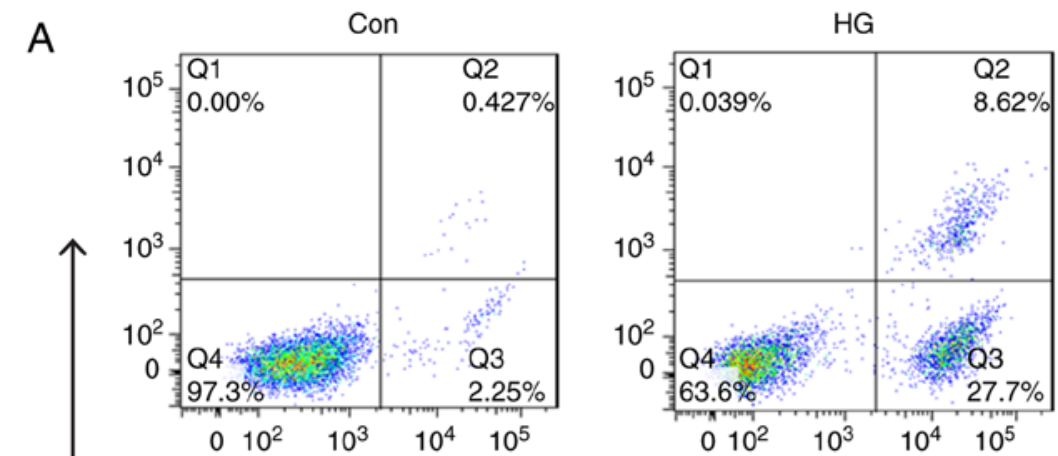

$\mathrm{Pl}$
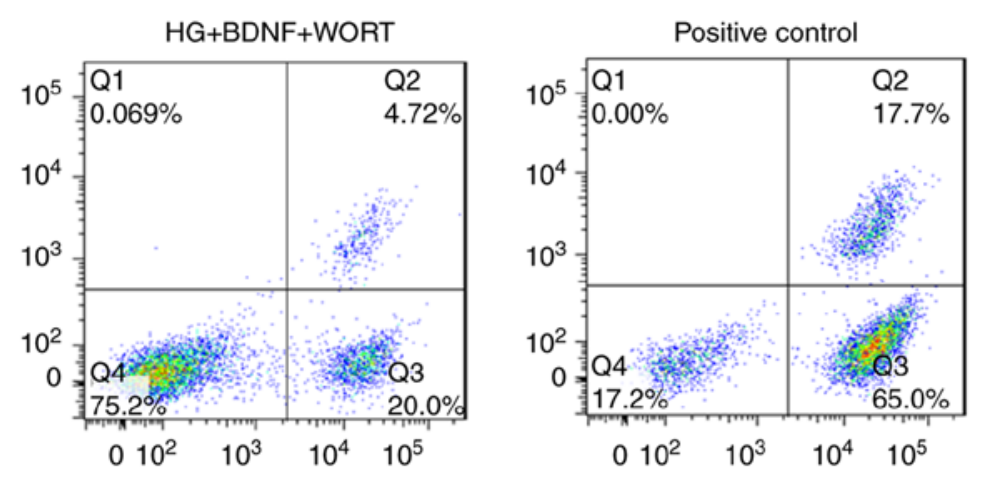

I
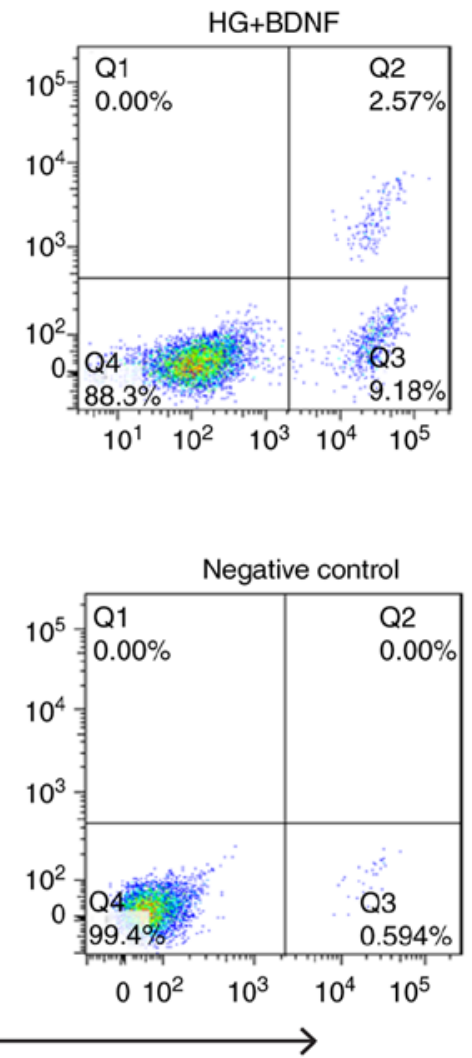

Annexin V-FITC

\section{B}

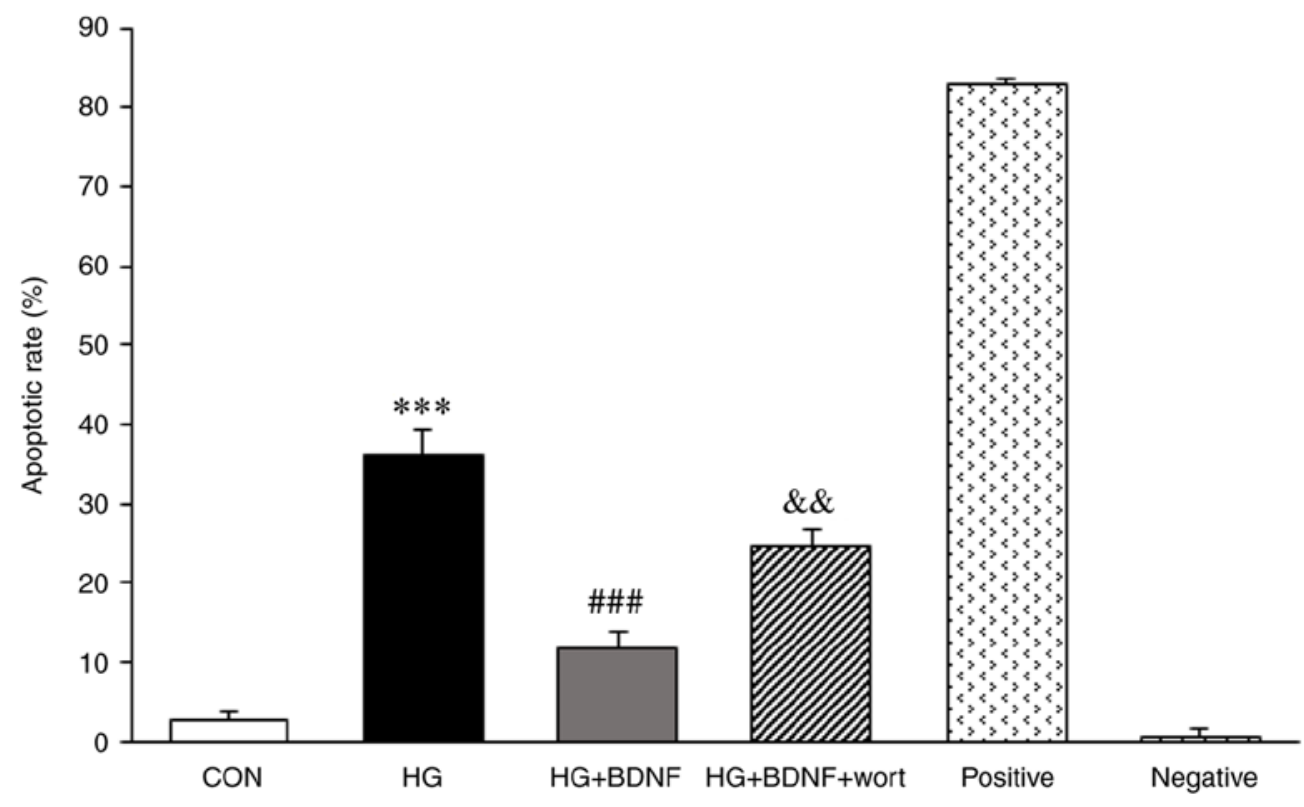

Figure 2. Effect of BDNF on HG-induced neuronal apoptosis. (A) Neuronal apoptosis was assayed by flow cytometry (Annexin V-FITC/PI staining). CON: $25 \mathrm{mM}$ glucose; HG: $75 \mathrm{mM}$ glucose for $72 \mathrm{~h}$; HG + BDNF: $50 \mathrm{ng} / \mathrm{ml}$ BDNF for $24 \mathrm{~h}$ followed by $75 \mathrm{mM}$ glucose for $72 \mathrm{~h}$; HG + BDNF + wort: $0.5 \mu \mathrm{M}$ wort pretreatment for $2 \mathrm{~h}$ to suppress PI3K, followed by $\mathrm{ng} / \mathrm{ml}$ BDNF for $24 \mathrm{~h}$ and then $75 \mathrm{mM}$ glucose for $72 \mathrm{~h}$. (B) Data are presented as the mean \pm standard deviation of three independent triplicate experiments. ${ }^{* * *} \mathrm{P}<0.001$, vs. CON group; ${ }^{\# \#} \mathrm{P}<0.001$, vs. HG group; \&\& $\mathrm{P}<0.01$, vs. HG $+\mathrm{BDNF}$ group. FITC, fluorescein isothiocyanate; PI, propidium iodide; CON, control; BDNF, brain-derived neurotrophic factor; HG, high glucose; wort, wortmannin.

CREB were significantly reduced on exposure to high glucose (all $\mathrm{P}<0.001$; Fig. 3A-C). BDNF significantly inhibited the effects of high glucose on the mRNA expression levels of Syn, Arc and CREB (all P<0.01; Fig. 3A-C). In addition, prior administration of wortmannin significantly attenuated the ability of BDNF to reverse the effects of high glucose on the mRNA expression levels of Syn $(\mathrm{P}<0.001)$, Arc $(\mathrm{P}<0.05)$ and
CREB ( $\mathrm{P}<0.01$; Fig. 3A-C). When the protein levels of Syn, Arc and CREB were assessed by western blotting (Fig. 4A-D), the results were consistent with those of the RT-qPCR experiments. Taken together, these data indicated that high glucose may lead to an imbalance in the synaptic plasticity of hippocampal neurons, and that this effect is suppressed by BDNF via PI3K signaling. 

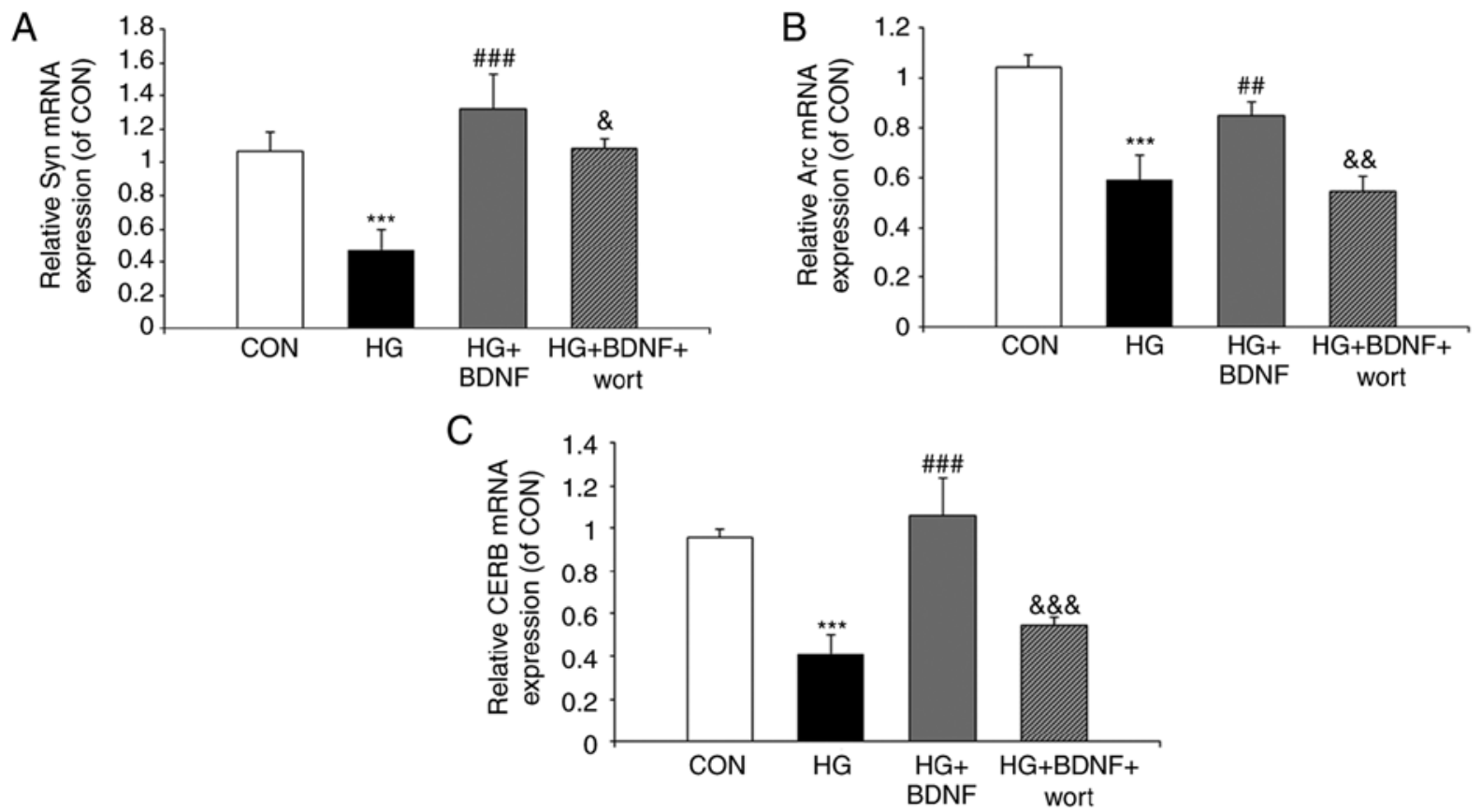

Figure 3. Effects of BDNF on the mRNA expression levels of synaptic plasticity-related proteins in primary hippocampal neurons under high glucose conditions. The mRNA expression of (A) Syn, (B) Arc and (C) CREB (C) in primary hippocampal neurons was monitored by reverse transcription-quantitative polymerase chain reaction analysis. GAPDH was used as an internal control. Data are presented as the mean \pm standard deviation of three independent experiments. ${ }^{* * *} \mathrm{P}<0.001$, vs. CON group; ${ }^{\# \#} \mathrm{P}<0.01$ and ${ }^{\# \# \#} \mathrm{P}<0.001$, vs. HG group; ${ }^{\&} \mathrm{P}<0.05$, \&\&\& $\mathrm{P}<0.01$ and ${ }^{\& \& \&} \mathrm{P}<0.001$, vs. HG + BDNF group. Syn, synaptophysin; CREB, cyclic AMP response element-binding protein; GAPDH, glyceraldehyde 3-phosphate dehydrogenase; CON, control; BDNF, brain-derived neurotrophic factor; HG, high glucose; wort, wortmannin.

A

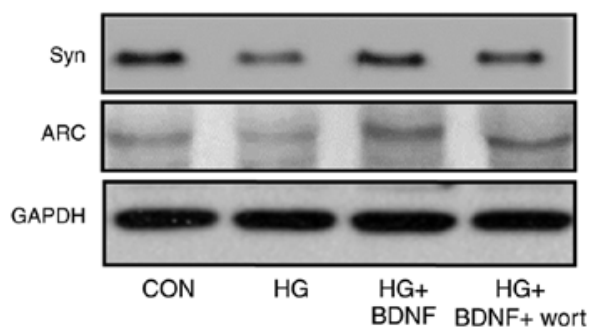

\section{CREB}

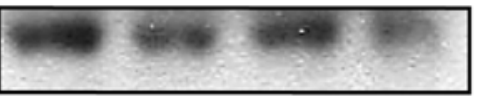

GAPDH

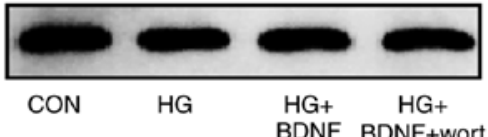

C

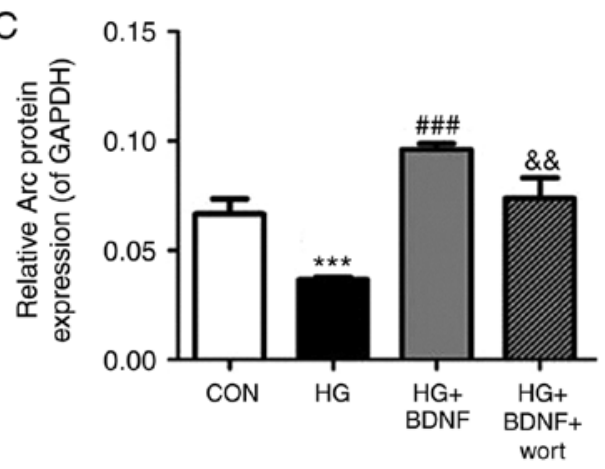

B

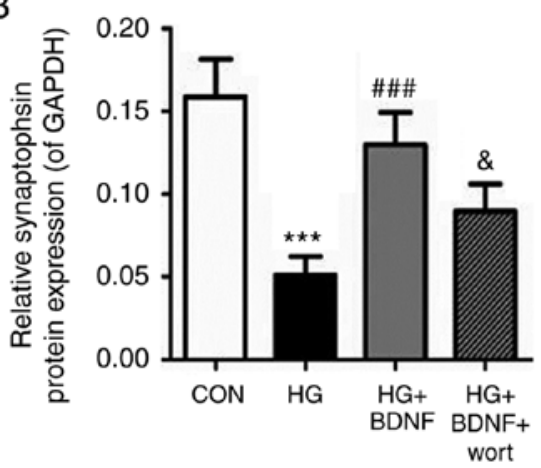

D

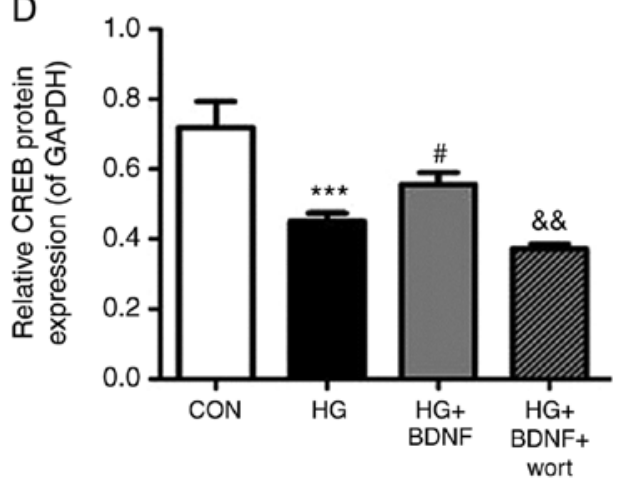

Figure 4. Effects of BDNF on the protein expression levels of synaptic plasticity-related proteins in primary hippocampal neurons under high glucose conditions. (A) Western blot analysis was used to measure the protein expression levels of (B) Syn, (C) Arc and (D) CREB in primary hippocampal neurons. GAPDH was used as an internal control. Data are presented as the mean \pm standard deviation of three independent experiments. ${ }^{* * *} \mathrm{P}<0.001$, vs. CON group; ${ }^{*} \mathrm{P}<0.05$ and ${ }^{\# \# \# /} \mathrm{P}<0.001$, vs. HG group; ${ }^{\&} \mathrm{P}<0.05$ and ${ }^{\& \&} \mathrm{P}<0.01$, vs. HG + BDNF group. Syn, synaptophysin; CREB, cyclic AMP response element-binding protein; GAPDH, glyceraldehyde 3-phosphate dehydrogenase; CON, control; BDNF, brain-derived neurotrophic factor; HG, high glucose; wort, wortmannin. 


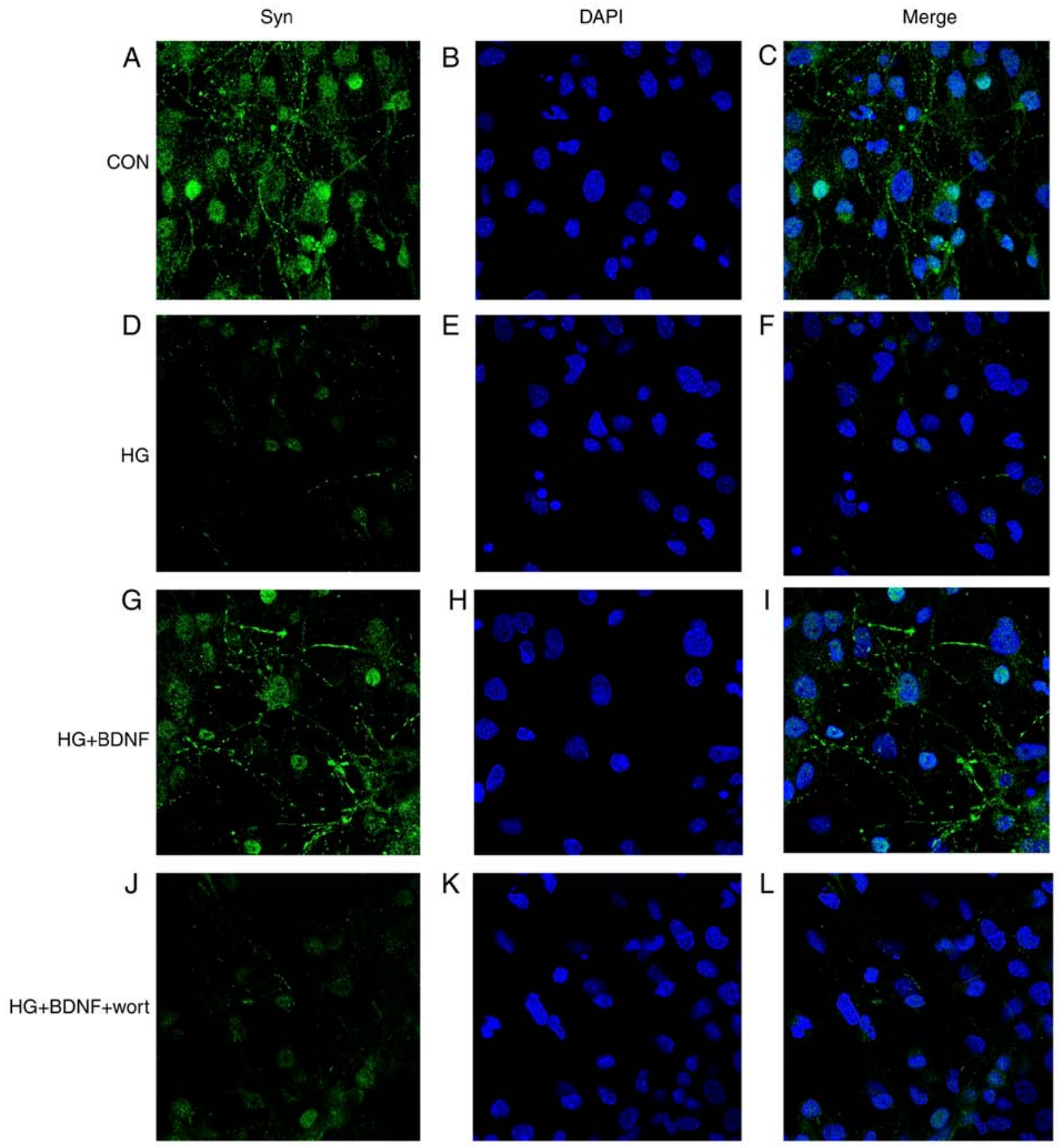

Figure 5. Effects of BDNF on the expression and distribution of Syn in primary hippocampal neurons under high glucose conditions. The expression and distribution of Syn were determined using immunofluorescence techniques. Syn is stained green, nuclei are stained blue with DAPI. (A) Syn, (B) DAPI and (C) Merge staining in the CON group; (D) Syn, (E) DAPI and (F) Merge staining in the HG group; (G) Syn, (H) DAPI and (I) Merge staining in the HG + BDNF group; (J) Syn, (K) DAPI and (L) Merge staining in the HG + BDNF + wort group. Magnification, x630. CON, control; BDNF, brain-derived neurotrophic factor; HG, high glucose; wort, wortmannin; DAPI, 4',6-diamidino-2-phenylindole.

$B D N F$ regulates the level and distribution of Syn in primary hippocampal neurons under high glucose conditions. The level and distribution of Syn can be used as an indirect measure of synaptic density (19). Therefore, the distribution of Syn in primary hippocampal neurons was assessed using immunofluorescence techniques. In the $\mathrm{CON}$ group, Syn protein was expressed in the neurites of hippocampal cells (Fig. 5A-C). However, the expression level of Syn was markedly reduced in hippocampal neurons in the HG group, with loss of expression in the neurites (Fig. 5D-F). BDNF normalized the levels and distribution of Syn protein in the neurons, and this effect of BDNF was prevented by wortmannin (Fig. 5G-L). These findings indicated that high glucose reduced synaptic density in primary hippocampal neurons, and that BDNF was able to reverse this effect via PI3K signaling.

BDNF upregulates protein expression levels of TrkB, Akt and p-Akt in primary hippocampal neurons under high glucose conditions. The expression levels of the downstream signaling proteins of BDNF were assessed to further investigate the molecular mechanisms underlying the neuroprotective effect of BDNF on hippocampal neurons under high glucose conditions (Fig. 6A). The protein expression levels of TrkB in the $\mathrm{HG}$ group were similar to those in the $\mathrm{CON}$ group, whereas 

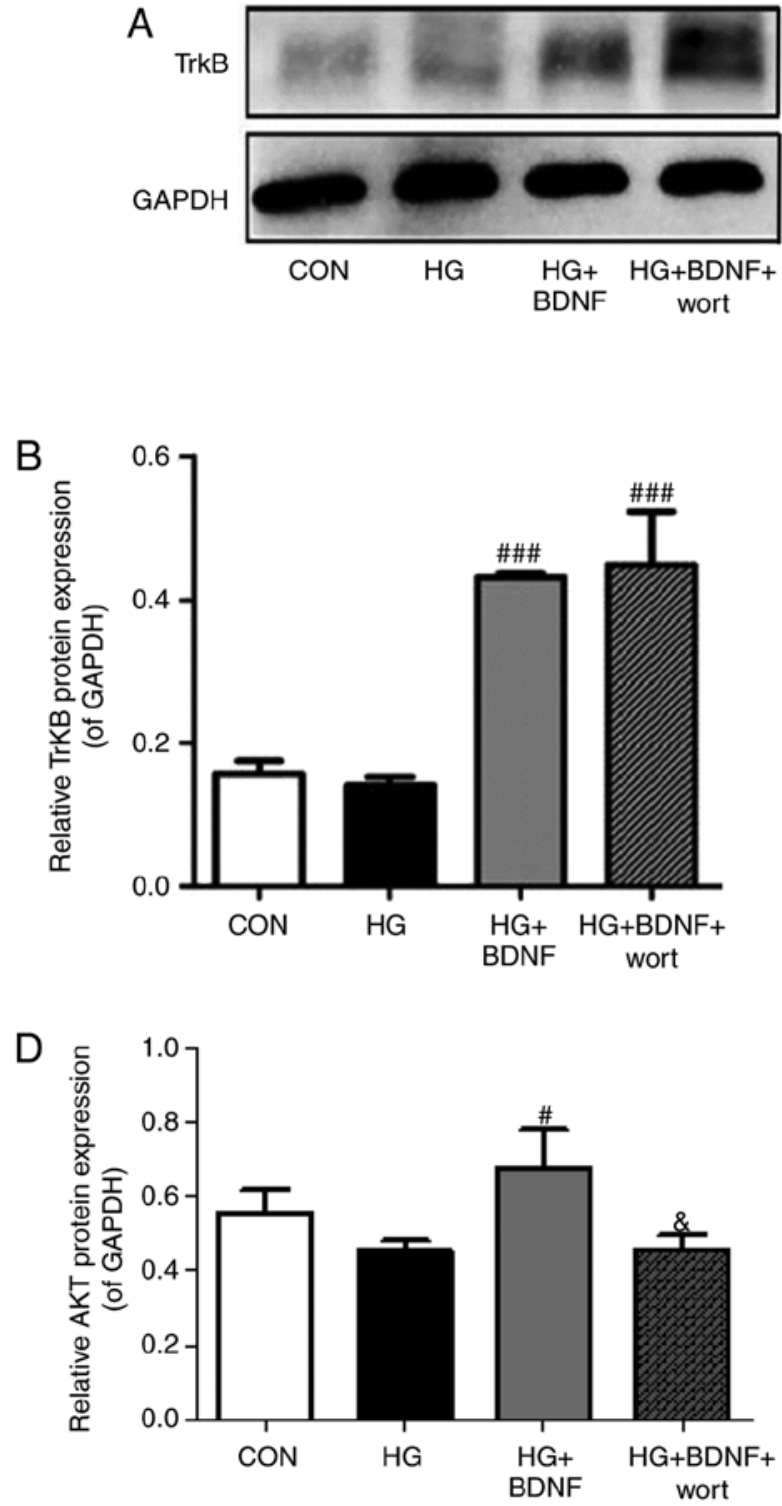
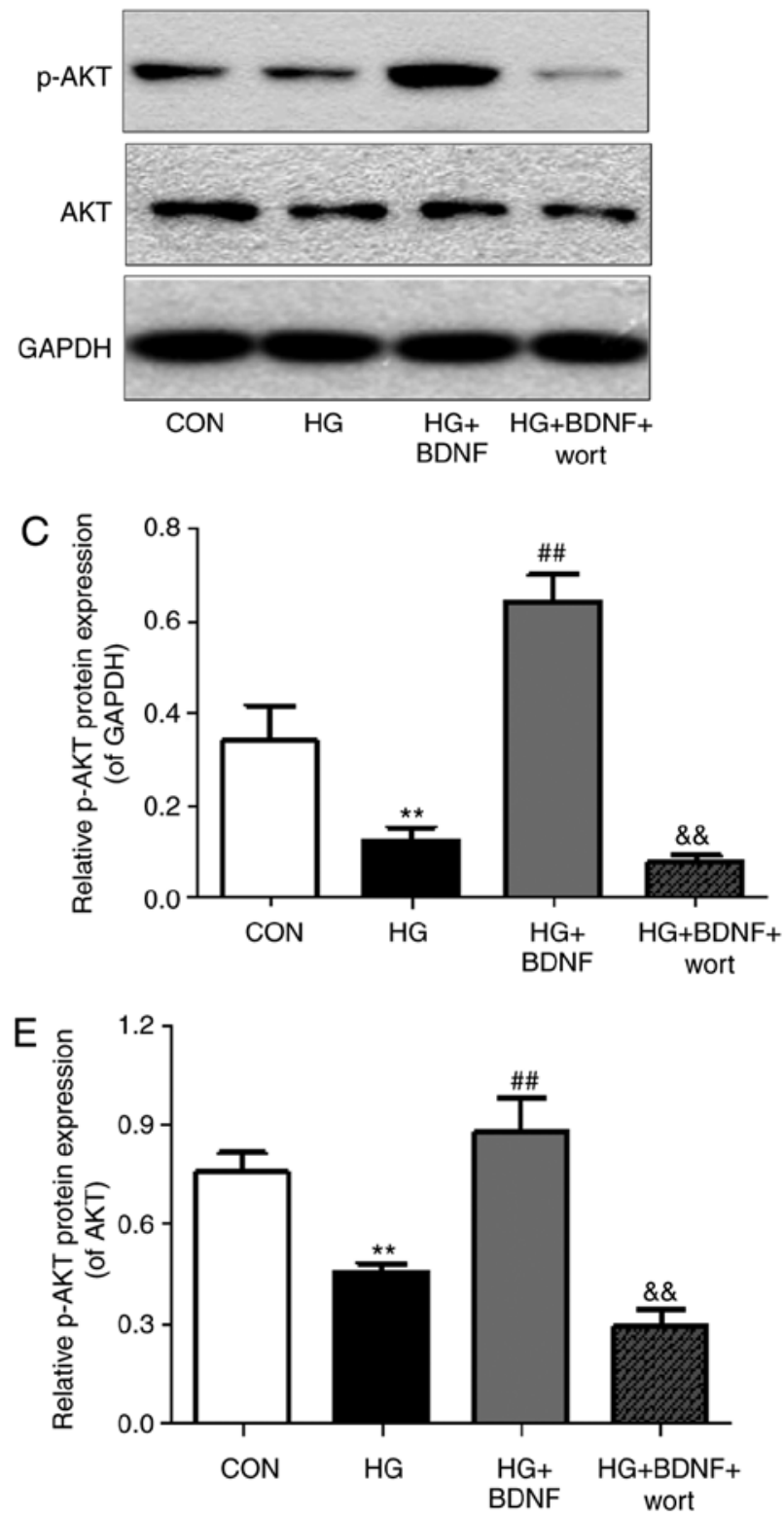

Figure 6. Effects of BDNF on protein expression levels of TrkB, Akt and p-Akt in primary hippocampal neurons under high glucose conditions. (A) Expression levels of TrkB, Akt and p-Akt were determined by western blot analysis, with data for (B) TrkB, (C) p-Akt, (D) Akt and (E) p-Akt/Akt presented as the mean \pm standard deviation of three independent experiments. ${ }^{* *} \mathrm{P}<0.01$, vs. CON group; ${ }^{\#} \mathrm{P}<0.05,{ }^{\# \#} \mathrm{P}<0.01$ and ${ }^{\# \# \#} \mathrm{P}<0.001$, vs. $\mathrm{HG}$ group; ${ }^{\circledR} \mathrm{P}<0.05,{ }^{\&} \mathrm{P}<0.01$, vs. HG + BDNF group. CON, control; BDNF, brain-derived neurotrophic factor; HG, high glucose; wort, wortmannin; TrkB, tropomyosin-related kinase B; p-, phosphorylated; GAPDH, glyceraldehyde 3-phosphate dehydrogenase.

combined treatment of $\mathrm{HG}+\mathrm{BDNF}$ resulted in increased protein expression levels of TrkB compared with those in the HG group $(\mathrm{P}<0.001$; Fig. 6B). The expression levels of TrkB were significantly increased in the $\mathrm{HG}+\mathrm{BDNF}+$ wort group compared with those in the HG group $(\mathrm{P}<0.001$; Fig. 6B), but did not differ significantly compared with those in the $\mathrm{HG}+\mathrm{BDNF}$ group. The expression levels of p-Akt, Akt, and p-Akt/Akt were significantly decreased in the $\mathrm{HG}+\mathrm{BDNF}+$ wort group compared with those in the $\mathrm{HG}+\mathrm{BDNF}$ group $(\mathrm{P}<0.01, \mathrm{P}<0.05$ and $\mathrm{P}<0.01$, respectively; Fig. $6 \mathrm{C}-\mathrm{E})$, whereas the $\mathrm{HG}+\mathrm{BDNF}$ group exhibited significantly higher protein expression levels of p-Akt, AKT and p-Akt/Akt compared with those in the HG group of cells $(\mathrm{P}<0.01, \mathrm{P}<0.05$ and $\mathrm{P}<0.01$, respectively; Fig. 6C-E). The expression levels of p-Akt and p-Akt/Akt were significantly decreased in the HG group compared with those in the CON group (both $\mathrm{P}<0.01$; Fig. $6 \mathrm{C}$ and $\mathrm{E}$ ), Taken together, these data suggested that high glucose may lead to abnormal plasticity in hippocampal neuronal synapses, which is reversed by BDNF via its cognate receptor TrkB and the downstream signaling protein Akt.

\section{Discussion}

In the present study, an in vitro model of rat hippocampal neurons was established from newborn rats, and neurite growth was evaluated by immunostaining for NeuN (39). Furthermore, hyperglycemic conditions were established for neuronal growth and it was demonstrated that BDNF protected against neuronal cell apoptosis induced by high glucose. In addition, BDNF increased the expression levels of 
synaptic plasticity-related proteins under high glucose conditions. Notably, the various effects of BDNF in rat hippocampal neurons treated with high glucose were dependent on PI3K-Akt signaling.

In the present study, BDNF protected neuronal cells from high glucose-induced apoptosis as demonstrated by flow cytometry. The neuronal apoptotic rate was decreased markedly (11.49, vs. $38.86 \%$ ) when the neurons were exposed to $75 \mathrm{mM}$ glucose for $72 \mathrm{~h}$ and administered with $50 \mathrm{ng} / \mathrm{ml}$ BDNF. A previous study demonstrated that the addition of BDNF prior to anoxia resulted in neuroprotective effects (40). The protective effect of BDNF was decreased following prolonged anoxia and irreversible neuronal injury. Therefore, in the present study, the hippocampal neurons were pretreated with BDNF for $24 \mathrm{~h}$ prior to their culture in the presence of high glucose, and a BDNF concentration of $50 \mathrm{ng} / \mathrm{ml}$ was selected based on the previous studies $(41,42)$. Consistent with the findings in the present study, Bathina et al (43) reported that RIN5F cells exhibited reduced viability following treatment with streptozotocin, which was reverted by BDNF.

The present study also demonstrated that the mRNA and protein expression levels of Syn, Arc and CREB were lower in hippocampal neurons exposed to high glucose than in neurons of the control group. Notably, the immunofluorescence experiments revealed a decrease in the protein expression of Syn in the neurites following treatment with high glucose, and this abnormal distribution of Syn was consistent with a reduction in synaptic density. These findings suggested that high glucose may lead to abnormal plasticity in hippocampal neuronal synapses via alterations in the levels, and thus functions, of proteins that are closely associated with synaptic plasticity. Consistent with these observations, Zhao et al (18) demonstrated that Syn was downregulated in primary neuronal cultures subjected to high glucose and hypoxia. Furthermore, a previous study demonstrated that the combination of hyperglycemia and hypoxia in mice resulted in cognitive impairment and was associated with significantly reduced protein levels of Syn in the hippocampus (19). It was suggested that the effects of high glucose and hypoxia on the protein levels of Syn may result from the enhanced degradation of Syn involving the E3 ubiquitin ligase, siah family (19). Another report found abnormal levels of certain synaptic proteins (synaptosomalassociated protein-25, synaptotagmin-1 and vesicular glutamate transporter-1) following long-term exposure of hippocampal neurons to hyperglycemia, suggesting that the trafficking of proteins to the synapse may be impaired (15).

In the present study, BDNF caused an increase in the mRNA and protein expression levels of Syn, Arc and CREB in hippocampal neurons treated with high glucose. BDNF also normalized the distribution of Syn in these cells. These observations suggested that the protective effect of BDNF on hippocampal neurons was achieved, at least in part, through enhancement of synaptic plasticity. Leal et al (44) demonstrated that BDNF can regulate hippocampal synaptic plasticity. In addition, a previous study found that rats fed on a high-fat, high-glucose diet to induce experimental diabetes exhibited impaired spatial learning, decreased hippocampal dendritic spine density and reduced long-term potentiation, and these changes were associated with a reduction in hippocampal BDNF levels (45). Arc has been demonstrated to exert a neuroprotective effect via decreased AMPA receptor current and glutamate receptor 2 internalization (46), therefore, the upregulation of Arc levels by BDNF may contribute to the neuroprotective effects of BDNF.

BDNF binds to TrkB and recruits proteins that activate several signal transduction cascades, including the sequential activation of insulin receptor substrate-1,PI3K and Akt (47). The BDNF signaling pathways activate CREB and CREB-binding protein, regulating the genes involved in neural plasticity (47). The PI3K-Akt signaling pathway is involved in synaptic plasticity, memory consolidation and synaptic morphogenesis $(48,49)$. In terms of the role of this pathway in diabetes, asiaticoside, a glycosylated triterpene from Centella asiatica, has been shown to attenuate diabetes-induced cognitive impairment and upregulate the expression of synaptic proteins via PI3K/Akt signaling (50). However, no previous reports have examined the role of the PI3K-Akt pathway in mediating the effects of BDNF in diabetic encephalopathy. In the present study, BDNF was demonstrated to activate PI3K-Akt signaling under high glucose conditions, as the levels of p-Akt and Akt were increased. In addition, BDNF enhanced the mRNA and protein expression levels of Arc, Syn and CREB, all of which can influence synaptic plasticity through the PI3K-Akt pathway as the effects of BDNF were inhibited by wortmannin. These findings indicated that BDNF-TrkB activates Akt under hyperglycemic conditions to reverse the abnormalities in synaptic plasticity and inhibit apoptosis. Taken together, these data indicated that BDNF protects hippocampal neurons partially through the upregulation of CREB and Arc, which is mediated through the PI3K-Akt signaling pathway.

In the present study, the expression of TrkB was increased following treatment with BDNF under high glucose conditions. Although it has been reported that the expression of TrkB is regulated by the cyclic AMP/CREB pathway in neurons (51), the administration of PI3K inhibitor did not decrease the expression level of TrkB, despite a reduction in CREB and p-Akt/Akt levels. This suggests that the regulation of the expression of TrkB by BDNF occurs upstream of Akt.

Of note, BDNF has also been demonstrated to protect retinal neurons from hyperglycemia via the TrkB/ERK/MAPK pathway and attenuate diabetic hyperglycemia via an insulin-independent mechanism in rats $(13,29)$. The regulation of long-term synaptic plasticity and memory formation by Arc are dependent on its phosphorylation by ERK protein, suggesting that MAPK kinases are important in the memory process (52). The findings of the present study indicate the possibility of potential interplay between the ERK/MAPK and PI3K-Akt pathways in the regulation of neuronal plasticity by BDNF.

In conclusion, the present study demonstrated that BDNF can activate the PI3K-Akt signaling pathway and induce the expressions of synaptic plasticity-related proteins in hippocampal neurons cultured under high glucose conditions. This improves synaptic plasticity in the hippocampal neurons and protects them from high glucose-induced apoptosis. These findings provide a theoretical basis for subsequent investigations on the mechanism of BDNF-mediated hippocampal neuroprotection. In addition, the present study provides novel insights into therapeutically targeting BDNF and PI3K-Akt signaling for the prevention of diabetic encephalopathy. 


\section{Acknowledgements}

Not applicable.

\section{Funding}

The present study was funded by the Shanghai Sixth People's Hospital Group Science Foundation, the Shanghai Science and Technology Commission Foundation Research Project (grant no. 13JC1401504) and the Chinese National Natural Science Foundation (grant no. 81300933).

\section{Availability of data and materials}

The datasets used and/or analyzed during the present study are available from the corresponding author on reasonable request.

\section{Authors' contributions}

YZ, YM and YTZ performed the experiments, were involved in data collection and drafted the manuscript. TH and QL performed the statistical analyses and were involved in study design. WL assisted in drafting the manuscript. All authors read and approved the final manuscript.

\section{Ethics approval and consent to participate}

All animal experiments were performed in accordance with the National Institutes of Health Guidelines for the Care and Use of Laboratory Animals and approved by the Ethics Committee of Animal Experiments of The Shanghai Sixth People's Hospital affiliated to Shanghai Jiao Tong University [permit no. SYXK (Shanghai) 2011-0128].

\section{Patient consent for publication}

Not applicable.

\section{Competing interests}

The authors declare that they have no competing interests.

\section{References}

1. Alam U, Asghar O, Azmi S and Malik RA: General aspects of diabetes mellitus. Handb Clin Neurol 126: 211-222, 2014.

2. Shekhar S, Wang S, Mims PN, Gonzalez-Fernandez E, Zhang C, He X, Liu CY, Lv W, Wang Y, Huang J and Fan F: Impaired cerebral Autoregulation-A common neurovascular pathway in diabetes may play a critical role in diabetes-related Alzheimer's disease. Curr Res Diabetes Obes J 2: pii: 555587, 2017.

3. Ninomiya T: Diabetes mellitus and dementia. Curr Diab Rep 14: 487, 2014.

4. Bitel CL,Kasinathan C,Kaswala RH,Klein WL and Frederikse PH: Amyloid- $\beta$ and tau pathology of Alzheimer's disease induced by diabetes in a rabbit animal model. J Alzheimers Dis 32: 291-305, 2012.

5. Sima AA: Encephalopathies: The emerging diabetic complications. Acta Diabetol 47: 279-293, 2010.

6. Chen S, Liu AR, An FM, Yao WB and Gao XD: Amelioration of neurodegenerative changes in cellular and rat models of diabetesrelated Alzheimer's disease by exendin-4. Age (Dordr) 34: 1211-1224, 2012.

7. Liu D, Zhang $\mathrm{H}, \mathrm{Gu} \mathrm{W}$ and Zhang M: Effects of exposure to high glucose on primary cultured hippocampal neurons: Involvement of intracellular ROS accumulation. Neurol Sci 35: 831-837, 2014.
8. Allen SJ, Watson JJ, Shoemark DK, Barua NU and Patel NK: GDNF, NGF and BDNF as therapeutic options for neurodegeneration. Pharmacol Ther 138: 155-175, 2013.

9. Noble EE, Billington CJ, Kotz CM and Wang C: The lighter side of BDNF. Am J Physiol Regul Integr Comp Physiol 300: R1053-R1069, 2011.

10. Jo YH and Chua SC Jr: The brain-liver connection between BDNF and glucose control. Diabetes 62: 1367-1368, 2013.

11. Nakagawa T, Tsuchida A, Itakura Y, Nonomura T, Ono M, Hirota F, Inoue T, Nakayama C, Taiji M and Noguchi $H$ : Brain-derived neurotrophic factor regulates glucose metabolism by modulating energy balance in diabetic mice. Diabetes 49: 436-444, 2000

12. Nonomura T, Tsuchida A, Ono-Kishino M, Nakagawa T, Taiji M and Noguchi H: Brain-derived neurotrophic factor regulates energy expenditure through the central nervous system in obese diabetic mice. Int J Exp Diabetes Res 2: 201-209, 2001.

13. Meek TH, Wisse BE, Thaler JP, Guyenet SJ, Matsen ME, Fischer JD, Taborsky GJ Jr, Schwartz MW and Morton GJ: BDNF action in the brain attenuates diabetic hyperglycemia via insulin-independent inhibition of hepatic glucose production. Diabetes 62: 1512-1518, 2013.

14. Sheng M, Sabatini BL and Südhof TC: Synapses and Alzheimer's disease. Cold Spring Harb Perspect Biol 4: pii: a005777, 2012.

15. Gaspar JM, Castilho A, Baptista FI, Liberal J and Ambrosio AF: Long-term exposure to high glucose induces changes in the content and distribution of some exocytotic proteins in cultured hippocampal neurons. Neuroscience 171: 981-992, 2010.

16. Masliah E, Terry RD, Alford M and DeTeresa R: Quantitative immunohistochemistry of synaptophysin in human neocortex: An alternative method to estimate density of presynaptic terminals in paraffin sections. J Histochem Cytochem 38: 837-844, 1990.

17. Tarsa L and Goda Y: Synaptophysin regulates activity-dependent synapse formation in cultured hippocampal neurons. Proc Natl Acad Sci USA 99: 1012-1016, 2002.

18. Zhao Y, Li Q, Jin A, Cui M and Liu X: E3 ubiquitin ligase Siah-1 downregulates synaptophysin expression under high glucose and hypoxia. Am J Transl Res 7: 15-27, 2015.

19. Li Q, Zhu XL, Jin AP, Liu XY and Zhao YX: Inhibition of synaptophysin ubiquitination may improve the intelligent drop due to high glucose and hypoxia. Int J Clin Exp Med 7: 5021-5030, 2014.

20. Lonze BE and Ginty DD: Function and regulation of CREB family transcription factors in the nervous system. Neuron 35: 605-623, 2002.

21. Middei S, Houeland G, Cavallucci V, Ammassari-Teule M, D'Amelio $M$ and Marie H: CREB is necessary for synaptic maintenance and learning-induced changes of the AMPA receptor GluA1 subunit. Hippocampus 23: 488-499, 2013.

22. Pugazhenthi S, Wang M, Pham S, Sze CI and Eckman CB: Downregulation of CREB expression in Alzheimer's brain and in A $\beta$-treated rat hippocampal neurons. Mol Neurodegener 6: 60, 2011.

23. Zhang N, Wen Q, Ren L, Liang W, Xia Y, Zhang X, Zhao D, Sun D, Hu Y, Hao H, et al: Neuroprotective effect of arctigenin via upregulation of P-CREB in mouse primary neurons and human SH-SY5Y neuroblastoma cells. Int J Mol Sci 14: 18657-18669, 2013.

24. Lacor PN, Buniel MC, Chang L, Fernandez SJ, Gong Y, Viola KL, Lambert MP, Velasco PT, Bigio EH, Finch CE, et al: Synaptic targeting by Alzheimer's-related amyloid beta oligomers. J Neurosci 24: 10191-10200, 2004.

25. Wegenast-Braun BM, Fulgencio Maisch A, Eicke D, Radde R, Herzig MC, Staufenbiel M, Jucker M and Calhoun ME: Independent effects of intra- and extracellular Abeta on learning-related gene expression. Am J Pathol 175: 271-282, 2009.

26. Dickey CA, Gordon MN, Mason JE, Wilson NJ, Diamond DM, Guzowski JF and Morgan D: Amyloid suppresses induction of genes critical for memory consolidation in APP + PS1 transgenic mice. J Neurochem 88: 434-442, 2004.

27. Ennis K, Dotterman H, Stein A and Rao R: Hyperglycemia accentuates and ketonemia attenuates hypoglycemia-induced neuronal injury in the developing rat brain. Pediatr Res 77: 84-90, 2015.

28. Liao GY, Li Y and Xu B: Ablation of TrkB expression in RGS9-2 cells leads to hyperphagic obesity. Mol Metab 2: 491-497, 2013.

29. Liu Y, Tao L, Fu X, Zhao Y and Xu X: BDNF protects retinal neurons from hyperglycemia through the TrkB/ERK/MAPK pathway. Mol Med Rep 7: 1773-1778, 2013. 
30. Xiang Q, Zhang J, Li CY, Wang Y, Zeng MJ, Cai ZX, Tian RB, Jia W and $\mathrm{Li} \mathrm{XH}$ : Insulin resistance-induced hyperglycemia decreased the activation of Akt/CREB in hippocampus neurons: Molecular evidence for mechanism of diabetes-induced cognitive dysfunction. Neuropeptides 54: 9-15, 2015.

31. Chen TJ, Wang DC and Chen SS: Amyloid-beta interrupts the PI3K-Akt-mTOR signaling pathway that could be involved in brain-derived neurotrophic factor-induced Arc expression in rat cortical neurons. J Neurosci Res 87: 2297-2307, 2009.

32. Kaech S and Banker G: Culturing hippocampal neurons. Nat Protoc 1: 2406-2415, 2006.

33. Chen Y, Cao CP, Li CR, Wang W, Zhang D, Han LL, Zhang XQ, Kim A, Kim S and Liu GL: Ghrelin modulates insulin sensitivity and tau phosphorylation in high glucose-induced hippocampal neurons. Biol Pharm Bull 33: 1165-1169, 2010.

34. Livak KJ and Schmittgen TD: Analysis of relative gene expression data using real-time quantitative PCR and the 2(-Delta Delta C(T)) method. Methods 25: 402-408, 2001.

35. Fath T, Ke YD, Gunning P, Götz J and Ittner LM: Primary support cultures of hippocampal and substantia nigra neurons. Nat Protoc 4: 78-85, 2009

36. Chen YJ, Huang XB, Li ZX, Yin LL, Chen WQ and Li L: Tenuigenin protects cultured hippocampal neurons against methylglyoxal-induced neurotoxicity. Eur J Pharmacol 645: 1-8, 2010.

37. Shao JL, Wan XH, Chen Y, Bi C, Chen HM, Zhong Y, Heng XH and Qian JQ: H2S protects hippocampal neurons from anoxia-reoxygenation through cAMP-mediated PI3K/Akt/p70S6K cell-survival signaling pathways. J Mol Neurosci 43: 453-460, 2011.

38. Chen WS, Yueh CY, Huang YA and Hwang E: An inverted method for culturing dissociated mouse hippocampal neurons. Neurosci Res 70: 118-123, 2011.

39. Boldrini M, Santiago AN, Hen R, Dwork AJ, Rosoklija GB, Tamir H, Arango V and John Mann J: Hippocampal granule neuron number and dentate gyrus volume in antidepressant-treated and untreated major depression. Neuropsychopharmacology 38: 1068-1077, 2013.

40. Luo XL, Mao M, Zhou H, Sun XM and Li SF: Neuroprotective effect of BDNF on hypoxia for embryonic rat cortical neurons in vitro. Sichuan Da Xue Xue Bao Yi Xue Ban 37: 373-377, 2006 (In Chinese).

41. Rankin SL, Guy CS, Rahimtula $M$ and Mearow KM: Neurotrophin-induced upregulation of p75NTR via a protein kinase C-delta-dependent mechanism. Brain Res 1217: 10-24, 2008.

42. Smith ED, Prieto GA, Tong L, Sears-Kraxberger I, Rice JD, Steward $\mathrm{O}$ and Cotman CW: Rapamycin and interleukin- $1 \beta$ impair brain-derived neurotrophic factor-dependent neuron survival by modulating autophagy. J Biol Chem 289: 20615-20629, 2014.
43. Bathina S, Srinivas N and Das UN: Streptozotocin produces oxidative stress, inflammation and decreases BDNF concentrations to induce apoptosis of RIN5F cells and type 2 diabetes mellitus in Wistar rats. Biochem Biophys Res Commun 486: 406-413, 2017.

44. Leal G, Afonso PM, Salazar IL and Duarte CB: Regulation of hippocampal synaptic plasticity by BDNF. Brain Res 1621: 82-101, 2015.

45. Stranahan AM, Norman ED, Lee K, Cutler RG, Telljohann RS, Egan JM and Mattson MP: Diet-induced insulin resistance impairs hippocampal synaptic plasticity and cognition in middle-aged rats. Hippocampus 18: 1085-1088, 2008.

46. Cohan CH, Stradecki-Cohan HM, Morris-Blanco KC, Khoury N, Koronowski KB, Youbi M, Wright CB and Perez-Pinzon MA: Protein kinase $\mathrm{C}$ epsilon delays latency until anoxic depolarization through arc expression and GluR2 internalization. J Cereb Blood Flow Metab 37: 3774-3788, 2017.

47. Bathina S and Das UN: Brain-derived neurotrophic factor and its clinical implications. Arch Med Sci 11: 1164-1178, 2015.

48. Garelick MG and Kennedy BK: TOR on the brain. Exp Gerontol 46: 155-163, 2011

49. Kennedy MB, Beale HC, Carlisle HJ and Washburn LR: Integration of biochemical signalling in spines. Nat Rev Neurosci 6: 423-434, 2005.

50. Yin Z, Yu H, Chen S, Ma C, Ma X, Xu L, Ma Z, Qu R and Ma S Asiaticoside attenuates diabetes-induced cognition deficits by regulating PI3K/Akt/NF-kB pathway. Behav Brain Res 292: 288-299, 2015.

51. Deogracias R, Espliguero G, Iglesias T and Rodriguez-Peña A Expression of the neurotrophin receptor trkB is regulated by the cAMP/CREB pathway in neurons. Mol Cell Neurosci 26: 470-480, 2004.

52. Nikolaienko O, Eriksen MS, Patil S, Bito $\mathrm{H}$ and Bramham CR: Stimulus-evoked ERK-dependent phosphorylation of activity-regulated cytoskeleton-associated protein (Arc) regulates its neuronal subcellular localization. Neuroscience 360: 68-80, 2017.

This work is licensed under a Creative Commons Attribution-NonCommercial-NoDerivatives 4.0 International (CC BY-NC-ND 4.0) License. 